\title{
Guest editorial to the special issue on "modeling: foundations and applications" (MODELS 2013)
}

\author{
Ana Moreira $^{1}$ - Bernhard Schätz ${ }^{2}$ - Peter Clarke ${ }^{3}$ - Antonio Vallecillo ${ }^{4}$
}

Received: 19 July 2015 / Accepted: 15 August 2015 / Published online: 12 November 2015

(C) Springer-Verlag Berlin Heidelberg 2015

\section{Introduction}

Since its beginnings, the use of models has always been a core principle in Computer Science. Recently, model-based engineering is gaining rapid popularity across various engineering disciplines. The pervasive use of models as the essential artifacts of the development process, and model-driven development of complex systems, has been strengthened by a focus on executable models and automatic transformations supporting the generation of more refined models and implementations.

Software models have become industrially accepted best practices in many application areas. Domains like automotive systems and avionics, interactive systems, business engineering, games, and Web-based applications commonly apply a tool-supported, model-based, or model-driven approach toward software development. The potential for early validation and verification, coupled with the generation of production code, has shown to cover a large percentage of

Ana Moreira

amm@fct.unl.pt

Bernhard Schätz

schaetz@informatik.tu-muenchen.de

Peter Clarke

clarkep@cis.fiu.edu

Antonio Vallecillo

av@lcc.uma.es

1 NOVA LINCS, Universidad Nova de Lisboa, Lisbon, Portugal

2 Fortiss and Technische Universität München, Munich, Germany

3 Florida International University, Miami, FL, USA

4 Universidad de Málaga, Málaga, Spain implemented functionality with improved productivity and reliability.

This increased success of using models in software and systems engineering also opens up new challenges, requiring collaborative research across multiple disciplines, ranging from offering suitable domain-specific modeling concepts to supporting legacy needs through models. The MODELS conference is devoted to model-based development for software and systems engineering, covering all types of modeling languages, methods, tools, and their applications.

The 2013 edition of the MODELS conference took place in the "magic" city of Miami, USA, from September 29 to October 4, 2013. The MODELS 2013 conference offered an opportunity for researchers, practitioners, educators, and students to come together, to reflect on and discuss our progress as a community, and to identify the important challenges still to be addressed and overcome.

In its sixteenth edition, the MODELS community was challenged to demonstrate the maturity and effectiveness of model-based and model-driven engineering and to explore their limits by investigating new application areas and combinations with other emerging technologies. This challenge resulted in 180 papers submitted to the MODELS 2013 Foundations (130) and Applications Tracks (50).

The Foundations Track papers provide significant contributions to the core software modeling body of knowledge in the form of new ideas and results that advance the state of the art. Two categories of Foundations Track papers were invited: technical papers, describing original scientifically rigorous solutions to challenging model-driven development problems, and exploratory papers, describing new, nonconventional modeling research positions or approaches that challenge the status quo and describe solutions that are based on new ways of looking at software modeling problems. 
The Applications Track papers demonstrate the relevance and effectiveness of the model-based paradigm of engineering. They include two categories of papers: application papers, providing a realistic and verifiable picture of the current state of the practice in model-based engineering and exploring the problems encountered by the industrial adoption of model-based techniques, and empirical evaluation papers, evaluating existing problem cases or scientifically validated proposed solutions through empirical studies, experiments, case studies, or simulations.

From the 180 submissions, 34 foundations and 13 applications papers were selected for presentation at the conference. Extended versions of the best papers, according to the feedback from the reviewers and their impact in the audience during the presentations, were invited for submission to a special issue in the SoSyM journal. The included papers not only indicate the current breadth, depth, and maturity of research and application of model-driven engineering, but also provide an overview of some of the current open issues and identify potential lines for further research.

\section{Selected papers for this special issue}

After the initial invitation, all selected papers underwent a thorough peer-review process, with at least two rounds of reviews for each paper. Following the journal guidelines and requirements, all submitted papers were refereed by at least three well-known experts in the field.

At the end of the review process, six papers were selected for this special issue:

- "A taxonomy of tool-related issues affecting the adoption of model-driven engineering," by Jon Whittle, John Hutchinson, Mark Rouncefield, Hakan Burden, and Rogart Hakan, discusses the impact of tools on model-driven engineering (MDE) adoption and practice. Tools have been usually blamed for the low uptake of MDE. The article revisits previous data on MDE used in industry and re-analyzes that data through the specific lens of MDE tools in an attempt to identify and categorize the issues that users have with the tools they adopted. New interviews are also conducted and analyzed though the same lens. Based on these studies, a taxonomy of technical, social, and organizational issues related to MDE tool use in practice is developed. This taxonomy serves as a checklist for companies developing and using tools and also points to a number of open challenges for those working on MDE tool development.

- "On the complex nature of MDE evolution and its impact on changeability," by Regina Hebig and Holger Giese, addresses the current lack of knowledge about evolution of MDE settings and its impact on changeability and other productivity dimensions. Through several case studies from different companies, it shows for the first time that evolution of MDE settings in practice occurs more frequently than expected and that its impact on the changeability of the evolving MDE settings can be quite significant.

- "Formal validation of domain-specific languages with derived features and well-formedness constraints," by Daniel Varro, Oszkár Semeráth, Ágnes Barta, Akos Horvath, and Zoltán Szatmári, addresses the problem of constructing design environments for complex domainspecific languages (DSL). Despite the wide range of existing tool support for this, it is still a tedious task because of the large number of derived features and well-formedness constraints complementing the domain metamodel that need to be handled. To tackle this problem, the paper proposes an automated mapping of EMF metamodels enriched with derived features and wellformedness constraints into an effectively propositional fragment of first-order logic that can be efficiently analyzed by back-end reasoners. The approach is supported by a tool that is used to show how a DSL from the avionics domain can be analyzed and validated. On the practical level, the paper also identifies and addresses relevant challenges and scenarios for systematically validating DSL specifications, also presenting initial performance experiments for the validation using Z3 and Alloy as back-end reasoners.

- "Language-specific model checking of UML-RT models" by Karolina Zurowska and Juergen Dingel introduces a verification method tailored to UML-RT, built around its main features such as hierarchical structures, action code, and asynchronous communication. The proposal departs from the prevailing trend in verification of model-driven development (MDD) models of translating them into an input language of one of the existing tools, most notably model checkers, to overcome some of their limitations. In particular, the input languages of model checkers are typically not compatible with MDD models, which can make the translations very complex and difficult to maintain; besides, it is more difficult to take advantage of specific features of the structure and semantics of models to, for example, speed up the analysis. The proposal enables very easy transformation of models and also reduces the necessary translations of verification results and directly supports the most important features of UML-RT.

- "Automated product line test case selection: case study and controlled experiment," by Shuai Wang, Shaukat Ali, Arnaud Gotlieb and Marius Liaaen, proposes an automated process for test case selection, aimed at reducing the overall effort required for this task, while achieving an acceptable level of the coverage of testing functionalities. The proposal is based on a systematic and automated 
methodology using a Feature Model for Testing that captures commonalities and variabilities of a product line and a Component Family Model for Testing that captures the overall structure of test cases in the repository. Several experiments show the gains in cost and performance (effectiveness and efficiency) of the automated methodology as compared with the manual approach.

- "Teaching model-driven engineering from a relational database perspective," by Don Batory and Maider Azanza, reinterprets MDE from the viewpoint of relational databases to provide an alternative way to understand, demonstrate, and teach MDE using concepts and technologies that should be familiar to undergraduates. Several application case studies and a user study serve to illustrate the viability and benefits of this interesting approach.

These papers contribute in different ways to the field of model-driven engineering, providing a deeper understanding of some of the issues that practitioners face when designing and developing non-trivial applications using models and model transformations, and present novel approaches, techniques, and tools for dealing with these issues.
Acknowledgements First, we would like to thank the authors of the papers of this special issue for accepting our invitation to extend their work and present their contributions here. Our gratitude also goes to the reviewers for the timely manner in which they assisted in choosing and making suggestions to improve the selected papers, and for their helpful and insightful comments. We are aware of all the time and effort involved in producing such constructive reports, and in helping authors improve their papers. Finally, we would like to show our appreciation to the SoSyM editorial office and in particular to Martin Schindler and Bernhard Rumpe, for their excellent support in the preparation of this special issue. 